\title{
Correction to: A Brush-Spin-Coating Method for Fabricating In Vitro Patient-Specific Vascular Models by Coupling 3D-Printing
}

\author{
Qing-Zhuo Chi, ${ }^{1}$ Li-Zhong Mu (i), ${ }^{1}$ Ying He, ${ }^{1}$ Yong Luan, ${ }^{2}$ and Yu-Chen Jing ${ }^{3}$ \\ ${ }^{1}$ Key Laboratory of Ocean Energy Utilization and Energy Conservation of Ministry of Education Affiliation, Dalian University \\ of Technology, Dalian 116024, China; ${ }^{2}$ Department of Anesthesiology, The First Affiliated Hospital of Dalian Medical \\ University, Dalian 116011, China; and ${ }^{3}$ Department of Vascular Surgery, The First Affiliated Hospital of China Medical \\ University, Shenyang 110001, China
}

(Published online 16 February 2021)

Correction to: Cardiovascular Engineering and

Technology (2020)

https://doi.org/10.1007/s13239-020-00504-9

This Erratum is to add additional funding information to the article.

This research was funded by the National Natural Science Foundation of China (Nos. 51976026, 11602053), Dalian Innovative Funding of Science and
Technology (No. 2018J12SN076), and The Fundamental Research Funds for the Central Universities DUT20GJ203.

The original article has been corrected.

Publisher's Note Springer Nature remains neutral with regard to jurisdictional claims in published maps and institutional affiliations.

The original article can be found online at https://doi.org/10. 1007/s13239-020-00504-9.

Address correspondence to Li-Zhong $\mathrm{Mu}$, Key Laboratory of Ocean Energy Utilization and Energy Conservation of Ministry of Education Affiliation, Dalian University of Technology, Dalian 116024, China.Electronic mail: qpdzkkk@mail.dlut.edu.cn; muliz@dlut.edu.cn; heying@dlut.edu.cn; cclyyly@163.com; 260522327@qq.com 